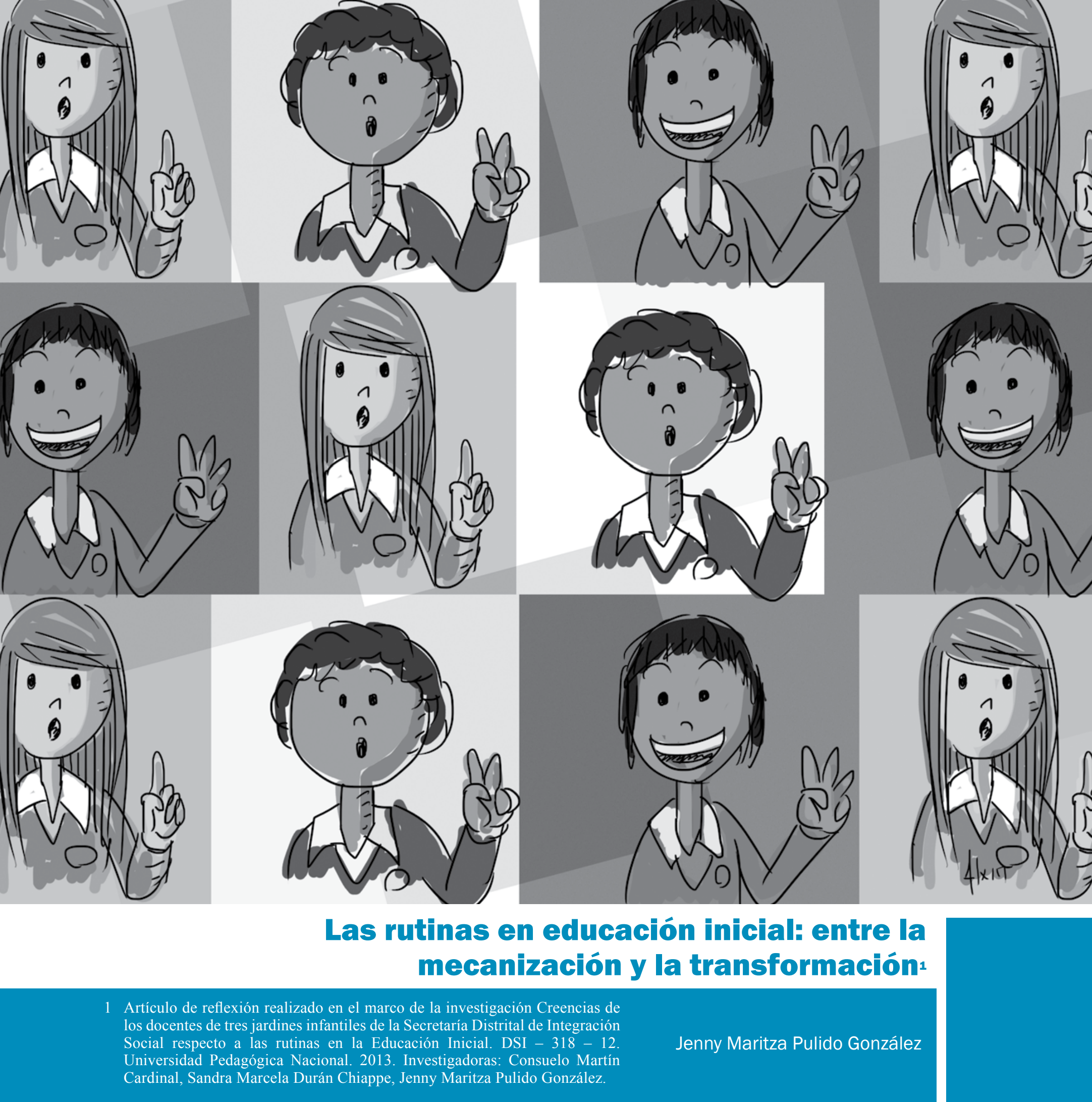


2 Docente Investigadora. Universidad Pedagógica Nacional. Magister en Educación; jepulido@pedagogica.edu.co

\section{Las rutinas en educación inicial: entre la mecanización y la transformación}

\section{Resumen}

En el contexto educativo y especialmente en la educación inicial pareciese que hablar de rutinas conlleva a desdeñar el aprendizaje fundamentado en intereses, necesidades y exploración autónoma del niño y la niña. Desde distintos lugares se ha advertido que la rutina atenta contra esto e impone una secuencia rígida de acciones mecanizadas e innegociables. No obstante, otros escenarios de enunciación la instalan como condición de posibilidad para la emergencia de nuevos procesos pedagógicos. En este contexto, el artículo presenta las tensiones que al respecto emergen en la educación infantil.

Palabras clave: rutinas, educación infantil, escuela, tiempo.

\section{The routines in initial education: between the mechanisation and the transformation}

\section{Summary}

In the educational context, and especially in initial education, it seems that talking about routines leads to rejecting the learning process based on interests, needs, and the child's self-exploration. Different voices have warned that the routine puts this at risk and imposes a rigid sequence of actions which are mechanical and non-negotiable. Nevertheless, other enunciation settings regard the routine as a condition that makes possible that new pedagogical processes arise. In this context, the article presents the tensions that due to that fact emerge in early education.

Keywords: Routines, education, infancy, school, time.

\section{As rotinas em educação inicial: entre a mecanización e a transformação}

\section{Resumo}

No contexto educativo, e especialmente, na educação inicial, parecesse que falar de rotinas implica a desdenhar a aprendizagem fundamentada em interesses, necessidades e exploração autónoma do menino e a menina. As vozes diferentes têm advertido que a rotina atenta contra isto e impõe uma sequência rígida de ações mecanizadas e innegociables. No entanto, outras definições de enunciação consideram a rotina de uma condição que torna possivel que surjam novos processos pedagógicos. Neste contexto, 0 artigo apresenta as tensões que ao respeito surgem na educação infantil.

Palavras-chave: rotinas, educação, infância, escola, tempo. 


\section{¿Qué lugares teóricos enriquecen las conceptualizaciones en relación con las rutinas?}

$\mathrm{L}$ as normas, en la vida de todos los grupos sociales, consuman un rol preponderante en tanto validan o sancionan prácticas que definen lo apropiado o no, $y$, en consecuencia, regulan las dinámicas de organización cotidiana de los colectivos humanos. La normalización se constituye por rutinas o normas electivas que pueden, con frecuencia, ser administradas por las personas.

"La normalización la entendemos como una resultante de la confluencia de diferentes rutinas operatorias habilitadas para configurar objetos o situaciones repetibles (caza, producción de flechas, símbolos lingüísticos) cuando en esta confluencia prevalecen, en una suerte de lucha por la vida, unas determinadas rutinas sobre las otras, también posibles, pero que quedan proscritas, vencidas o marginadas, sin perjuicio de que sigan siendo siempre virtualmente realizables, frente a la rutina victoriosa" (García Sierra, 1990, p. 235).

Ello significa que las normas son consideradas como rutinas triunfantes en el avance de la humanidad, por lo que pueden entenderse como piedra angular en la evolución y, en consecuencia, de los desarrollos sociales, científicos, económicos, políticos y culturales de la misma. La conducta normada, como las rutinas, las ceremonias o las acciones ritualizadas, diferencian al hombre de los demás animales, por cuanto estructuran la vida humana vía lenguaje y dinamizan los procesos de transmisión, acopio y transformación de las conductas, tradiciones, prácticas culturales y demás modos de actuar de las personas en tiempos y contextos determinados.

Ello presupone que la rutina es susceptible de ser gestionada en razón a los fines que se persigan, al ideal que se considera oportuno obtener, o a la admisión de prácticas que, al ser naturalizadas, se acostumbran ejecutar. Dicha idea de gestión cobra especial importancia en tanto traduce la rutina en potencial dinamizador cargado de un importante valor simbólico - en ritual-, o por el contrario, en acciones que en el día a día se acostumbran y por tanto, no exigen el detenerse a pensar en el sentido y significado de las mismas.

\section{Las rutinas como instrumento de neutralización}

Para profundizar en este aspecto, resulta preciso remitirse a los procesos de modernización pedagógica por los cuales atravesó el país durante el siglo XX y, de manera especial, a las campañas de higienización de la población, pues allí puede situarse, en gran medida, el afán por neutralizar, a través de diferentes acciones y dispositivos de control a los sujetos, y así, dejar atrás el pasado indígena, pobre e ignorante que además de resultar vergonzoso, era una amenaza latente para el anhelado progreso.

Las ideas de formar un hombre moderno ejercieron gran poder sobre los imaginarios acerca de la niñez. Álvarez (2002, p. 36 ), sostiene que en este contexto inició la proliferación de instituciones preocupadas por la infancia, entre las que se encuentra la escuela, institución que cumpliría un papel protagónico al convertirse en la mejor alternativa para corregir y prevenir los peligros a los que constantemente se veían enfrentados los niños en una época de urbanización, pero también, para minimizar los defectos individuales y colectivos. Podría decirse que el énfasis puesto en el cambio de hábitos populares por hábitos refinados, se consideraba uno de los peldaños necesarios de transitar para la consecución de un pueblo civilizado.

La escuela comenzó a constituirse en escenario idóneo para la transmisión de los ideales de progreso y civilización, por lo que el maestro, el médico y los intelectuales cercanos a los discursos médico-biológicos se convirtieron en protagonistas de la formación de la infancia, no para hacer de la higiene un componente educativo más, sino, más allá de eso, para constituirla en eje organizador de las rutinas escolares.

El higienizar, entendido como la "vigorización y restauración de la población, particularmente de la infancia" (Noguera, 2002, p. 284), se constituyó en propósito para el logro de un pueblo culto, civilizado y, en consecuencia, cercano al ideal europeo, por lo que prácticas como la limpieza, la buena alimentación y el vestuario, ocuparon un lugar privilegiado con respecto a la educación. Sanear, limpiar, se convirtieron en acciones, más importantes, o por lo menos urgentes, que la educación. A decir de Noguera: "la higiene, como condición empírica y como referencia teórica, se constituyó en la base 
sobre la cual debía girar toda la dinámica de la vida escolar" (Noguera, 2002, p. 284).

De cara a la higiene intelectual, física y moral, tiempos, espacios, cuerpos y mentes, fueron dispuestos según el criterio de médicos e higienistas, de manera tal que fuese posible controlar a la infancia, y de esta manera, asegurar un futuro próspero, o por lo menos, no tan indecoroso para la nación.

\section{"Para la distribución del tiempo se tendrá en cuenta la naturaleza de las clases, dejando de preferencia para las primeras horas aquellas más difíciles y para las últimas las que, como el dibujo, la historia, etc., requieren menor esfuerzo; Chabot propone tres clases en la mañana: dos abstractas y una lección concreta, y dos en la tarde: una lección concreta y un ejercicio, con un trabajo cotidiano de 6 horas, entre los 7 y los 12 años, de 7 horas entre los 12 y los 15 , y de 8 horas para los mayores de esta edad" (Manual escolar, citado por Noguera, 2002, p.281).}

Se observa pues, la organización de los contenidos escolares de cara a las disposiciones de médicos e higienistas. Administrar el tiempo instauró maneras específicas de actuar en la escuela y, en correspondencia con ello, de clasificar a los niños según sus edades y habilidades. La rutinización se configuró en piedra angular para ocupar las mentes y los cuerpos, para hacerlos útiles, para neutralizar la infancia y liberarla de su incompletud.

Saldarriaga (2003, p. 172), sostiene que la infancia comenzó a considerarse, fuertemente, como una etapa de la evolución humana, como el período de desarrollo de las funciones adaptativas de la especie y, en consecuencia, el más frágil y susceptible a toda clase de enfermedades e influencias del contexto. De este modo, se vuelve la mirada a la protección de la infancia como aspecto esencial para la higiene mental que: "permitiría corregir a tiempo la taras de la población. Se establecieron los consultorios prenupciales, los prenatales, las salas de maternidad y se dio énfasis al ejemplo y a la educación" (Herrera, 1999, p. 106). La idea que regía tales prácticas, consideraba que una mala educación transformaría los individuos en anormales o delincuentes, mientras que una educación bien dirigida los haría sanos y útiles.

Es en este escenario, en el cual, para Herrera (1999, p. 108), cobra especial importancia el papel de la madre y la maestra, ellas se consideraban fundamentales para transmitir la idea de conciencia sanitaria, las buenas costumbres, el amor, los de-

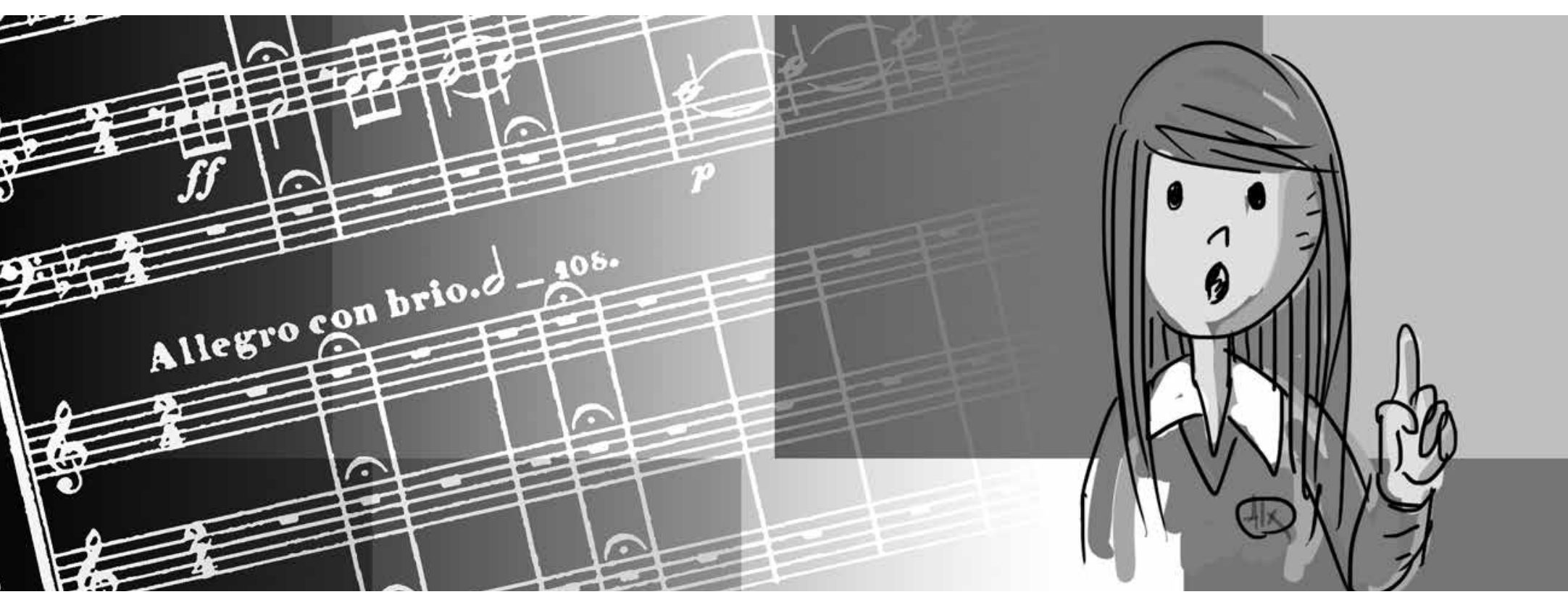

84 Educación y Ciudad N²4 Enero - Junio de 2013 ISSN 0123-0425 PP. 81-92 


\section{Ya no se trataba de la rigidez y, por qué no decirlo, arbitrariedad de la llamada "escuela tradicional"; ya el poder no se ejercía en y sobre los cuerpos, ahora, interesaba la formación de una clase dirigente"}

beres ciudadanos, lo cual, naturalmente, requería de espacios, tiempos y lugares destinados para ello, y además, para dar solución al problema de la vagancia y del trabajo infantil que, para la época, ya eran considerados obstáculos en la consecución del ideal de un pueblo culto y patriótico capaz de asegurar la transmisión de las ideas nacionalistas a las futuras generaciones.

Por su parte, la Escuela Activa, junto a la influencia de los discursos de la medicina, la biología y la psicología, expresados entre otros por Jean Piaget, María Montessori, Ferrèire, Decroly, Dewey y Henri Binet, instaló una concepción de disciplina que, aunque se distanciaba de la relación vertical maestro alumno y en cambio apostaba por el "descubrimiento" del niño (a), de sus intereses y necesidades, encontró nuevos mecanismos para, a decir de Saldarriaga (2002), "poner la disciplina al servicio de la vida" (p. 175).

"La idea de la disciplina implica o supone idea de orden, de armonía, de razón. Establece una conformidad con las leyes de la vida y la naturaleza. Cuando hay disciplina, hay progreso realizado, división del trabajo, sentido de la jerarquía y concentración individual. En una palabra, es una aspiración a la unidad y la armonía. Desde el punto de vista social, ella supone el espíritu de colaboración, solidaridad y armonía; desde el punto de vista individual, cohesión, rectitud, dominio de sí mismo, armonía entre sentimientos, la inteligencia y la acción” (Saldarriaga, 2003, p. 176).

El afán moderno por optimizar tiempos y espacios encontró su lugar bajo esta concepción de disciplina que perseguía la productividad y aprovechamiento de las mentes, los cuerpos y las actividades de los individuos. Así, según Saldarriaga: "la autoridad se justifica, no por una jerarquía de sumisión sino por la necesidad científica de unidad del proceso, de clasificación y jerarquización funcional- técnica” (2003, p.176).

La escuela se dispuso, entonces, en un dispositivo para obtener, a través de la administración de los sujetos, de sus cuerpos, tiempos y espacios, un modelo cercano al proyecto nacionalista, pero también a un sistema de jerarquías sociales en el que se privilegiaron los alcances particulares que las características biológicas y psicológicas permitiesen. Así pues, ya no se trataba de la rigidez y, por qué no decirlo, arbitrariedad de la llamada "escuela tradicional"; ya el poder no se ejercía en y sobre los cuerpos, ahora interesaba la formación de una clase dirigente, "de los más capaces", lo cual, naturalmente, señalaba la dificultad de los pobres para desarrollar sus capacidades intelectuales. Este panorama cristalizó la idea de la rutina como eslabón entre la educación del cuerpo y una infancia aséptica que asegurase el control sobre los sujetos:

\begin{abstract}
"La disposición en serie de las actividades sucesivas permite toda una fiscalización de la duración por el poder: posibilidad de un control detallado y de una intervención puntual (de diferenciación, de corrección, de depuración, de eliminación) en cada momento del tiempo [...] El poder se articula directamente sobre el tiempo; asegura su control y garantiza su uso" (Foucault, 2004, p. 164).
\end{abstract}

Es importante advertir que gran parte de estas ideas de saneamiento y control estructuran -aunque no sea de manera consiente- muchas de las prácticas que hoy en día se reflejan en algunas instituciones escolares y en las que las normalizaciones, los estilos examinables y cuantificables, revisten de significado la rutina, asumiéndola como la organización rígida seguida por los maestros que busca, fundamentalmente, la disciplina y el control.

\section{Las rutinas: entre el medio y el fin}

La premura por preparar a niños y niñas para los momentos posteriores de su vida, ha sido un propósito que difícilmente pasa inadvertido por maestros y maestras de educación inicial. El afán por procurar un óptimo espacio que garantice el éxito o, por lo menos, la adaptación de los más pequeños a sus futuras experiencias, moviliza buena parte de las estrategias y apuestas de trabajo de los docentes. 


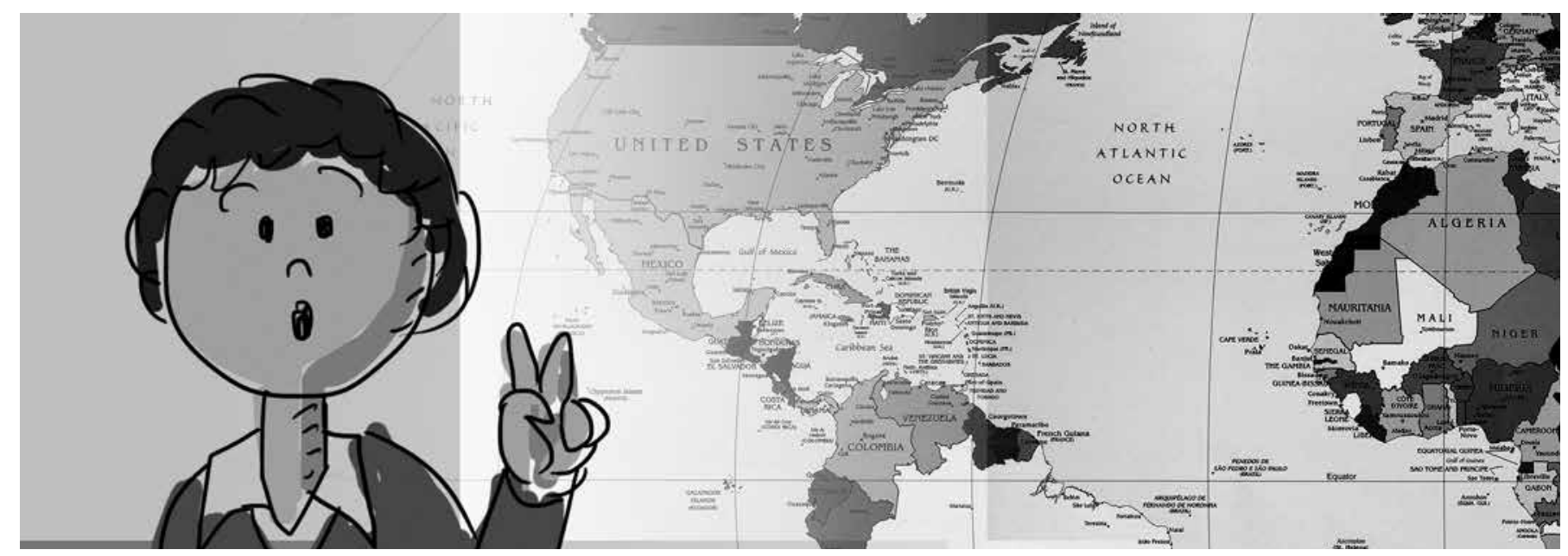

A ello puede agregársele el acento en su protección, en su bienestar físico, de manera concreta, en la satisfacción de algunas de sus necesidades básicas, acento que en algunos contextos se convierte en propósito y fin único del trabajo con niños y niñas de educación inicial. Se trata entonces de intentar suplir las carencias de su núcleo familiar, así, es pues un trabajo sustentado en las privaciones, en las ausencias, en las necesidades del otro, en este caso, de la infancia que asiste a estos escenarios.

En el caso del acompañamiento a los bebés, el tema del asistencialismo se torna incluso más complejo. En amplios sectores de la sociedad se ha fortalecido la idea de que con ellos sólo se puede trabajar alrededor de la satisfacción de sus necesidades básicas, tarea que fácilmente puede desempeñarla cualquier mujer. No es gratuito que el porcentaje más alto de docentes en educación inicial sean mujeres, fundamentalmente, porque se considera que su figura representa características propias para el cuidado y la protección de la infancia. La historia muestra cómo en Colombia, hacia mediados del siglo XX, aparecen textos donde la mujer es asumida básicamente como madre; por tanto, como la responsable de proteger a la infancia y de ofrecerle los parámetros y principios morales sobre los que va a crecer $y$ formarse.

Ello se suma que la formación de hábitos, la idea casi exclusiva de preparar para la adaptación al entorno, y a la vez para la sujeción a la norma, a la regla, a los tiempos y espacios impuestos, atraviesa buena parte de las prácticas a las que un gran porcentaje de niños y niñas pueden acceder. En ámbitos así, regularmente, el medio se transforma en fin, y entonces lo preponderante en el día a día con los más pequeños es, fundamentalmente, cuidarlos, alimentarlos y mantenerlos limpios. Estas tres acciones configuran el día a día de la institución.

Las rutinas se ubican en el escenario de lo mecánico, de las acciones desprovistas de mayor reflexión, de improvisación ${ }^{3}$, pues, al ser tan rígidas, se reduce la posibilidad de espontaneidad. Todo está claro, todo está mecanizado, todo está dicho. Como ya se expresó, dichas rutinas organizan las demás actividades del jardín infantil: las propuestas pedagógicas inician cuando ya todos están limpios y culminan cuando la comida se ha servido. Las rutinas de aseo, y especialmente de alimentación, ocupan gran parte del tiempo de niños y niñas; todo cesa, todo puede ser después, excepto la comida.

Pero, ¿a qué obedece el marcado énfasis en la alimentación e higiene de los más pequeños?, ¿es gratuito que gran parte de la jornada de las instituciones de educación inicial se destine a ello?

Rodríguez sostiene que, de mano con el naciente interés de los escolasnovistas por la educación y de cara a la incidencia de la medicina en este campo:

\footnotetext{
3 Asumiendo los momentos de improvisación como espacios de creatividad e imaginación.
} 


\begin{abstract}
"El trabajo médico se centró, por una parte, en la higiene y la prevención, que se establecieron como problemáticas cruciales debido a las exiguas condiciones de salubridad que mantenían tasas de mortalidad infantil desmedidas, y por otro, en el diagnóstico, clasificación y tratamiento de enfermedades infantiles que se hicieron visibles a propósito del establecimiento de la escuela obligatoria" (Rodríguez 2003, p. 32).
\end{abstract}

De este modo, la prevención comenzó a ocupar un lugar preponderante en los discursos de médicos y educadores. La nutrición se constituyó en factor esencial para la salubridad de la población y naturalmente, para las campañas de higienización expuestas anteriormente. Ahora bien, el documento Nutrición en Colombia, estrategias de país 2011-2014, elaborado por el Banco Interamericano de Desarrollo, sostiene que:

\begin{abstract}
"La situación nutricional de una población refleja en gran medida su nivel de bienestar y puede ser utilizada para identificar inequidades que podrían afectar su productividad. Así, la desnutrición crónica, identificada por el retraso en el crecimiento lineal o la talla baja de los niños, está asociada con menor desempeño escolar, menor productividad y menor ingreso laboral en la vida adulta" (Neufeld, Rubio, Pinzón y Tolentino, 2010, p. 5).
\end{abstract}

Tal afán por el fortalecimiento de la vida vía alimentación, favorece también la incorporación del orden social: "por medio de la repetición de sabores determinados, se introducen normas de conducta que, a un mismo tiempo, reglamentan una población y, potencializan el cuerpo de cada individuo en particular" (Richter A.C. y Fernández Vaz, A. 2008, p. 92).

Ciertamente, el contenido mismo de tales prácticas pasa como inadvertido por muchos maestros, pues hace parte de un interés legítimo por el bienestar de niños y niñas que no tendría sentido poner en cuestión, pero sí, comprender a profundidad.

\footnotetext{
“Junto a las prácticas higiénico-alimenticias destinadas a los pequeños comensales, encontramos algunas de las relaciones del proceso de civilización, con la organización de la sociedad contemporánea, con los procesos de individualización y totalización, o por lo menos, con su proyecto social interesado, sobretodo, en el cuerpo (y en la) de la especie. Los
}

momentos de higiene y alimentación de la/en la guardería nos parecen revelar algunas formas de circulación de saberes relacionados a 'una organización normal y colectiva (y, por lo tanto, política) de la vida humana"” (Agamben, 2004, citado por Richter A. C. y Fernández Vaz, 2008)”.

Desde este ángulo, las prácticas asistencialistas en los primeros años de vida del ser humano, contribuyen enormemente a la internalización de pautas socio culturales en contextos específicos, por lo cual, los distanciamientos de dichas posturas y la puesta en marcha de acciones de carácter pedagógico, impactan con mayor fuerza en escenarios dispuestos a reflexionar con respecto a la norma, el orden, la rutinización.

Es evidente que la preocupación por la nutrición, la higiene y el cuidado infantil resulta apenas obvia y necesaria, no obstante, cuando dichas inquietudes ocupan el lugar del propósito, cuando el medio se transforma en fin y las actividades del día a día se centran en ello exclusivamente, la rutina es despojada de su potencial renovador y, en cambio, se le ubica en el lugar de la repetición, al cual ha sido confinada por muchos de los defensores del trabajo basado en intereses y necesidades de niños y niñas.

\section{Las rutinas: ¿Momentos que se repiten sin sentido o posibilidades para el maestro y desarrollo para el niño y la niña?}

Desde la perspectiva de autores como Rimoli (2008), o Fabres (2006), en la vida cotidiana de las instituciones de educación inicial se viven momentos organizados en secuencia, que se repiten día tras día sin modificación y sentido alguno, se hacen lo más rápido posible, sin cesar. El momento de saludo, la comida, el paso de una actividad a otra, se vuelven mecánicos tanto para los maestros como para los niños. Según Fabres (2006), muchas veces se consideran estas actividades como incomodas y monótonas.

Desde esta vivencia, las rutinas no son objeto de planeación y se convierten en un asunto de hábitos de orden, aseo, alimentación y comportamiento. Según Rimoli y Ros (2009), estas prácticas rutinarias, consolidadas por la interacción de los sujetos a través del tiempo, corresponderían a un mecanismo de 


\section{Las rutinas, entonces, pueden convertirse en la oportunidad para el pleno ejercicio de la autonomía, en tanto se generen espacios para la toma de decisiones"}

control social y disciplinamiento, relacionados con una determinada cultura escolar, esencia de todo proceso de institucionalización. Esto se logra por medio de un entrenamiento donde el niño incorpora determinados comportamientos socialmente válidos a través de la repetición de canciones, versos o la espera de una recompensa. Así, las rutinas como mecanismo de control social, poseen elementos distintivos:

\begin{abstract}
"Suelen repetirse sin ser pensados como propuesta de aprendizaje para los niños, por lo cual tampoco se despliega en su desarrollo una situación didáctica. Se asocian a formas de control y organización externas de la conducta del niño. Parecen contraponerse a lo que se denomina 'actividades centrales' o 'actividades programadas', con una valoración inferior” (Rimoli y Ros, 2009, p. 97).
\end{abstract}

Ante este panorama, se propone el gran desafío de pensar y vivir las rutinas como una oportunidad para el maestro y un espacio-tiempo privilegiado para el desarrollo del niño. En este sentido, se apuesta por cambiar estas acciones en experiencias cotidianas significativas que marcan la manera como el niño se percibe así mismo. Esto supone por parte del docente una reflexión crítica frente a su labor diaria y una comprensión de todos los momentos y espacios de la jornada como fundamentales. Así, hay que:

"Pensar en la recuperación de estos espacios como terrenos fértiles para el trabajo pedagógico, transfórmalos en objetos de conocimientos y plantearse interrogantes acerca de ellos. Preguntarse sobre el sentido y significado de las acciones que realizamos cada día, supone crear condiciones favorables para el aprendizaje de los alumnos y para la revitalización del trabajo institucional" (Rimoli M del C. y Ros N., 2009, p. 98).

Reconocer las rutinas como contextos de aprendizaje, supone evidenciar algunas de sus funciones que, según Zabalza (1996), constituyen, en primera instancia:
"Un marco de referencia que una vez aprendido por el niño, da una gran libertad de movimientos tanto a los niños como al profesor: provee una especie de estructuración mental que permite dedicarse y dedicar sus energías a lo que se está haciendo sin pensar en lo que vendrá después. En cuanto marco constante permite además al profesor introducir en él cualquier tema, asunto o actividad novedosa que hay surgido inesperadamente. De esta manera lo nuevo entra a funcionar como contenido o material de las rutinas diarias" (p.173).

Las rutinas, entonces, pueden convertirse en la oportunidad para el pleno ejercicio de la autonomía, en tanto se generen espacios para la toma de decisiones, la participación y la resolución de problemas. Todo esto enmarcado en un respeto por los tiempos diferenciales de los niños. Consideradas así, las actividades reportan un sinnúmero de beneficios para el desarrollo infantil, como lo proponen Carmé Thió-Pep y Llenas (2007):

"Las entradas y salidas son situaciones cuyo contenido educativo reside fundamentalmente en el aprendizaje de habilidades y actitudes relativas a la capacidad de relación social, de comunicación entre personas de distintas edades y de distinta procedencia. Son situaciones que permiten ampliar el conocimiento de uno mismo, de los sentimientos, y emociones propias y de los demás, y adquirir recursos para avanzar en el camino de la socialización, de la autonomía y del equilibrio emocional" (p. 72).

Así, en el momento de la comida y las situaciones de higiene entran en juego sus afectos, su autoestima, el sentimiento de sentirse respetado por el otro. En la medida que se le dé un lugar al niño en estas acciones cotidianas, va a lograr una mayor comprensión de su finalidad y tomará la iniciativa para su desarrollo. La regularidad y ritualidad: "en los momentos de atención, libera a los niños de ansiedades e incertidumbres y así mismo favorece su protagonismo en estos contextos de experiencia" (Fortunati, 2006, p. 147). 


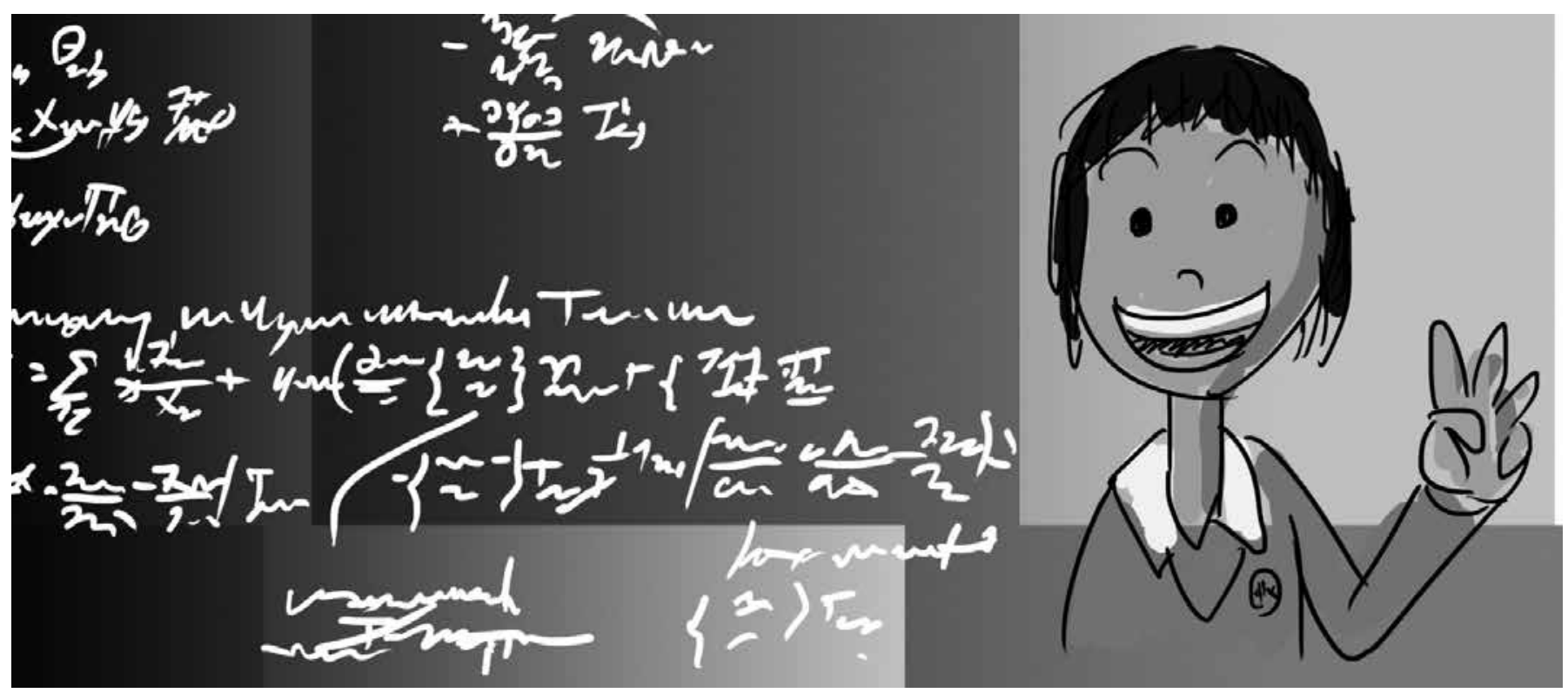

Todo esto significa que las rutinas se pueden revalorizar en un marco de regularidad, mas no de repetición mecánica, como posibilidades pedagógicas flexibles que juegan un papel preponderante en la Educación de la Primera Infancia.

Es importante considerar los momentos de cuidado como momentos de encuentro privilegiado entre adultos y niños, en los que se respeta y se escuchan las demandas y reacciones de estos últimos, ajustando así la respuesta a sus ritmos, gustos y necesidades. De esta manera, el niño, según Fabrés (2006), no será objeto de una atención rápida, sino que se aprovechará esta relación personalizada para establecer relaciones de complicidad sólidas y estables: "si reorganizamos el tiempo de la jornada, concediendo más importancia al tiempo de las rutinas, el ambiente se volverá más amable, más tranquilo, más placentero, de intercambio y diálogo, donde el valor fundamental será de respeto por el niño" (p. 17).

\section{El tiempo en la Educación Inicial}

La manera como se organiza el tiempo en educación inicial, es un tema presente en los documentos oficiales, en los textos referidos a este nivel y en distintas investigaciones que se han realizado alrededor de la cuestión de la educación en los pri- meros años. Así, se exponen ejemplos de posibles distribuciones del horario escolar, y del sentido del tiempo para el niño y para el educador, como la importancia del establecimiento de rutinas.

Dentro algunos de estos planteamientos, se pone de presente que las rutinas o formas de organizar los tiempos, son un reflejo de la apuesta pedagógica de la institución y marcan una manera de concebir al sujeto y sus interacciones: “(...) la organización de los tiempos educativos refleja unos supuestos psicopedagógicos, una jerarquía de valores, unas formas de administrar la escuela, en definitiva, una cultura escolar" (Viñao, 1998, p. 130, retomado por Vaca, 2008).

La vida diaria en los jardines infantiles se establece en función de unos intereses y principios que marcan la manera como el niño se desenvuelve en la institución, determinan unos énfasis, tanto en la secuencia como en la duración de los momentos. Expresa cuál es la prioridad de la institución; muchas veces, la organización de los tiempos está determinada por unos principios de carácter empresarial y eficientista, en lo que se quiere es "ganar tiempo", un tiempo productivo versus un tiempo evolutivo, la pregunta es entonces: 


\section{La sociedad competitiva del momento ejerce una influencia en el ámbito educativo: es una carrera contra el tiempo que emprenden educadores y niños y muchas veces los padres también"}

\begin{abstract}
“¿Cómo adaptar la organización del centro a las características de las edades, en lugar de aplicar criterios organizativos estándar para todas las edades sin tener en cuenta las características evolutivas específicas y reconocidas?" (Red territorial de educación infantil de Cataluña, 2009, p.79).
\end{abstract}

Se plantean unos horarios rígidos que no se corresponden con los tiempos reales de los niños, por lo que surge así la pregunta sobre: ¿cómo ajustar los tiempos personales con los tiempos del grupo y con los tiempos de la organización?

En educación inicial, el tema del tiempo está directamente ligado con la manera como se concibe al niño y en qué medida se considera que tiene derecho a su tiempo. En las propuestas pedagógicas se observa, en ocasiones, una preocupación porque el niño haga las cosas rápido y bien. Así, la sociedad competitiva del momento ejerce una influencia en el ámbito educativo: es una carrera contra el tiempo que emprenden educadores y niños y muchas veces los padres también, parece haber un afán por mostrar resultados y productos, pero ¿será lo más pertinente para el niño en desarrollo?, ¿qué tanto gusto se le puede extraer a las experiencias pedagógicas, cuando hay que terminar cuanto antes, cuando hay que acabar lo que se esté haciendo, pues sencillamente el tiempo demanda momentos determinados para cada actividad?

El tiempo no se puede perder, por eso frecuentemente las experiencias ligadas al juego se ven como una pérdida de tiempo: "En ese jardín pierden el tiempo, el niño sólo va a jugar".

De alguna manera, la cultura escolar muestra en el manejo del tiempo, el control que se ejerce sobre las acciones del maestro y, en consecuencia, sobre el actuar de niños y niñas.

Es por tanto un tema importante para reflexionar y estudiar, como lo expone la investigación sobre los ritmos de la infancia de Cabanellas, Eslava y otros (2007), la cual lleva a compren- der cómo en las instituciones de educación inicial, el tiempo es el tiempo del adulto y, en la jornada con sus momentos, se induce a un cierto control y habituación a factores externos; principalmente en lo que tiene que ver con la alimentación y el aseo, se crea una cierta regularidad que es igual para todos los niños, sin importar sus particularidades:

\begin{abstract}
"En nuestra investigación sobre el fluir temporal de las actuaciones infantiles han surgido temas de discusión que afectan directamente a las programaciones escolares. Generalmente, son los adultos los que pretenden determinar el ritmo temporal de las experiencias infantiles al introducir pautas de organización que rompen la actividad, unificando tiempos para todos los niños. Rompiendo los ritmos infantiles en la necesidad de articular un orden interno en la marcha de la escuela, que es necesario para una buena organización escolar, se rompen también los necesarios tiempos de la infancia. Este es un problema real que el educador debe resolver con la sensibilidad necesaria para conjugar ambos tiempos" (Cabanellas, Eslava y otros, 2007, p. 40).
\end{abstract}

Ahora bien, el tiempo y su organización son una parte fundamental de la labor del docente; él planea su acción pedagógica con un sentido e intencionalidad, y al estudiar la jornada escolar en su organización se muestran particularidades en la forma cómo se concibe la educación y sus componentes. Este es el caso del estudio de Vaca (2008), quien analizó la jornada escolar en centros de educación infantil para apreciar los diferentes significados que va adquiriendo el cuerpo a lo largo de la jornada, encontrando que:

"La estructura que se construye procura diversificar la presencias corporales, de tal modo que den como resultado una curva respetuosa con los ritmos y tiempos biológicos, psicológicos y sociales del alumnado, la tranquilidad, sosiego y previsibilidad que supone la repetición de las acciones educativas, y el orden en el que se van produciendo 
a lo largo del día, de la semana, no sólo no merman, sino que facilitan la creatividad y la autonomía” (p. 39).

Estos hallazgos invitan a mirar un poco más allá el manejo de los horarios, en tanto que sus estructuras guardan, como se señaló, diferentes concepciones sobre la Educación inicial y elementos centrales como el cuerpo, el juego y el lugar de las rutinas de cuidado.

En este mismo sentido, y recurriendo de nuevo a Vaca (2008), se encuentran las razones por las cuales el horario semanal tiene un gran interés en las actividades profesionales: "Pone en evidencia los acuerdos y diferencias entre el horario oficial y el horario real, expresa los modos de entender y proceder de los maestros y maestras en la complejidad de la vida del aula, manifiesta en su conjunto el modo de entender y desarrollar la profesión docente en la práctica" (p. 49).

Por su parte, Paniagua y Palacios (2008), refiriéndose a la estructura de las actividades en educación infantil, proponen que debe haber un equilibrio y flexibilidad, es conveniente una secuencia estable de actividades que les permitan los niños predecir lo que viene. Los autores, hacen un especial énfasis en los tiempos de espera que en las instituciones se convierten en momentos caracterizados por el desorden, los conflictos y el aburrimiento.

Es preciso buscar alternativas para los niños que hayan terminando, cuidar los tiempos de preparación de los materiales, involucrar a los niños en los preparativos, y tener en cuenta que no todos deben estar haciendo lo mismo al mismo tiempo. Esto permite un respeto a la diversidad de los ritmos e intereses, garantizando, según los autores, la autonomía. En este mismo sentido, Puig Rovira (2001) (retomado por Vaca 2008), expresa que:

"La vida en un aula escolar, y en cualquier otra institución social, ha de estar pautada por un conjunto de rutinas que hagan previsibles la relación personal y el trabajo. La seguridad que buscamos será la consecuencia de la pre- visibilidad. Advertimos, sin embargo, que seguridad y previsibilidad no irán en detrimento de valores como la creatividad, la autonomía y la democracia, sino que son condiciones que los hacen posibles" (p. 72).

Es así como es posible reconocer en una estructura temporal estable pero no rígida, el encuadre necesario para moverse con seguridad, creatividad y autonomía.

En el marco expuesto, las rutinas no pueden asumirse como estructuras inmóviles e inflexibles que sólo buscan reproducir unos saberes, unos contenidos, unas maneras de ser y estar en mundo, sino que por el contrario, pueden entenderse como un proceso que sostiene la construcción en los primeros años de una identidad que no es premeditada, sino construida a partir de su propia experiencia en interacción con el entorno, los objetos, sus pares y maestros y maestras, pues vale la pena decir que en la rutina el niño y la niña no responden automáticamente a unos momentos pensados para organizar la jornada, sino que se implica en ellos, construyendo esquemas mentales y movilizando estructuras de pensamiento que les permiten comunicarse, explorar, jugar.

Finalmente, no se puede negar que en Educación Inicial, las rutinas están articuladas con los hábitos, los cuales reflejan los contenidos de la cultura en los que están inmersos y, a su vez, proyectan maneras particulares de actuar, desde éstos, el niño y la niña construyen significados. La rutina y el hábito constituyen rituales que sin lugar a dudas se convierten en oportunidades de negociación y contextualización del devenir cultural, social e histórico.

En ese sentido, estudiar la rutina se convierte en una posibilidad que el maestro puede y debe considerar para pensar las prácticas que configuran la cultura escolar $\mathrm{y}$, de este modo, transformarlas, pero no desde el exterior, sino desde la reflexión de la comunidad educativa, pues sólo desde allí dejarán de ser asumidas como imposiciones y reflejo de dominación y control, para ser pensadas desde los símbolos y significados que expresan intencionalidades pedagógicas. 


\section{Referencias}

Álvarez Gallego, A. (2002). "Los niños de la calle. Bogotá 19001950”. En Historia de la educación en Bogotá, V. 2. Bogotá: IDEP, Alcaldía Mayor de Bogotá.

Cabanellas, I., Eslava, J. J y otros. (2007). Ritmos Infantiles: tejidos de un paisaje interior. Barcelona: Octaedro.

De Pol, C. y Llenas, P. (2007). Pequeños y competentes: de las actividades cotidianas al aprendizaje de la autonomía. En Planificar la etapa de 0-6. Compromiso de sus agentes y práctica cotidiana. Barcelona: Grao.

Fabrés, M. (2006 Noviembre-Diciembre). En el día a día nada es banal, nada es rutina. En $I N-F A N-C I A, 100$, p. 17.

Fortunati, A. (2006). La Educación de los niños como proyecto de la comunidad. La experiencia de San Miniato. Barcelona: Editorial Octaedro.

Foucault, M. (2004). Vigilar y castigar. Buenos Aires: Siglo XXI Editores.

García Sierra, Pelayo. (1990). Diccionario filosófico. Obtenido el 3 de julio de 2012, desde http://www.filosofia.org/filomat/df235.htm

Guillen, C. I. (2008). Los rituales escolares en la escuela pública polimodal Argentina. Obtenido el 10 de Septiembre de 2011, desde http://www.scielo.org.ar/scielo.php?script=sci_arttext\&pi$\mathrm{d}=\mathrm{S} 185116942008000100008 \& \operatorname{lng}=\mathrm{es} \& \mathrm{nrm}=$ iso

Herrera, M. C. (1999). Modernización y escuela nueva en Colombia. Bogotá: Editorial P \& J.

Lynnette, N., Rubio M., Pinzón L. y Tolentino, L. (2010). Nutrición en Colombia, estrategias de país 2011-2014. Bogotá: Banco Interamericano de Desarrollo.
Paniagua, G. y Palacios, J. (2008). Educación Infantil: Respuesta educativa a la diversidad. Madrid: Alianza editorial.

Red Territorial de Educación Infantil de Cataluña. (2009). La educación de 0-6 años hoy. Barcelona: Octaedro.

Richter, A. C., y Fernández, V. A. (2008). Alimentación e higiene en la rutina de la educación infantil: calidad de vida como artificio en la instrucción de las conductas y potencialización de los hijos de la nación. Educación física y ciencia, 10. Obtenido el 26 de Septiembre de 2011, desde http://www.memoria.fahce.unlp.edu.ar/art_revistas/pr.3698/pr.3698.pdf.

Rimoli, M. y Ros, N. (2009). Rutinas y situaciones didácticas. En Rutinas y rituales en Educación Inicial. Cómo se organiza la vida cotidiana. Buenos Aires: Ediciones Novedades Educativas.

Rimoli, M. (2008). Juego, rutinas e indagaciones guiadas. En El juego en la Educación Infantil. Buenos Aires: Novedades Educativas.

Rimoli, M. y Roth, S. (2008). Prácticas de rutina, actos y recreaciones. Trabajo presentado en el Congreso Repensar a Niñez en el Siglo XXI, Mendoza, Argentina.

Rodríguez, S. (2003). La educación para la primera infancia en Iberoamérica. Pedagogía y Saberes, 19. Bogotá: Universidad Pedagógica Nacional, 32.

Saldarriaga, O. (2003). El oficio del maestro. Prácticas y teorías de la Escuela Nueva en Colombia. Bogotá: Editorial Magisterio.

Zabalza, M. (1996). Didáctica de la educación infantil. Madrid: Narcea. 